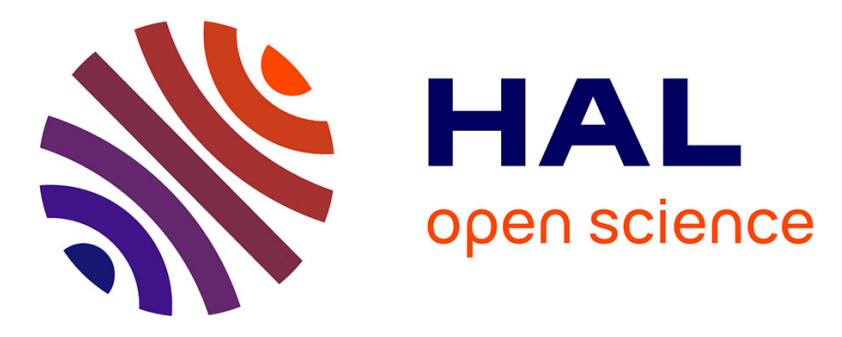

\title{
Compression of high-energy ultrashort laser pulses through an argon-filled tapered planar waveguide
}

\author{
Shihua Chen, Amélie Jarnac, Aurélien Houard, Yi Liu, Cord L. Arnold, Bing
}

Zhou, Benjamin Forestier, Bernard Prade, André Mysyrowicz

\section{To cite this version:}

Shihua Chen, Amélie Jarnac, Aurélien Houard, Yi Liu, Cord L. Arnold, et al.. Compression of highenergy ultrashort laser pulses through an argon-filled tapered planar waveguide. Journal of the Optical Society of America B, 2011, 28 (5), pp.1009. 10.1364/JOSAB.28.001009 . hal-00852002

HAL Id: hal-00852002

https://hal-polytechnique.archives-ouvertes.fr/hal-00852002

Submitted on 19 Aug 2013

HAL is a multi-disciplinary open access archive for the deposit and dissemination of scientific research documents, whether they are published or not. The documents may come from teaching and research institutions in France or abroad, or from public or private research centers.
L'archive ouverte pluridisciplinaire HAL, est destinée au dépôt et à la diffusion de documents scientifiques de niveau recherche, publiés ou non, émanant des établissements d'enseignement et de recherche français ou étrangers, des laboratoires publics ou privés. 


\title{
Compression of high-energy ultrashort laser pulses through an argon-filled tapered planar waveguide
}

\author{
Shihua Chen, ${ }^{1,2}$ Amelie Jarnac, ${ }^{1}$ Aurélien Houard, ${ }^{1}$ Yi Liu,,${ }^{1}$ Cord L. Arnold,${ }^{3}$ \\ Bing Zhou, ${ }^{1}$ Benjamin Forestier, ${ }^{1}$ Bernard Prade, ${ }^{1}$ and André Mysyrowicz ${ }^{1, *}$ \\ ${ }^{1}$ Laboratoire d'Optique Appliquée, ENSTA, Ecole Polytechnique, CNRS UMR 7639, Palaiseau 91761, France \\ ${ }^{2}$ Department of Physics, Southeast University, Nanjing 211189, China \\ ${ }^{3}$ Department of Physics, Lund University, PO Box 118, SE-221 00 Lund, Sweden
}

(Dated: February 14, 2011)

\begin{abstract}
We propose a hollow tapered planar waveguide for compression of high-energy ultrashort laser pulses. Direct measurements suggest that it seems to find a very good trade-off among the energy throughput, the beam focusability, and the pulse compressibility. With a Ti:sapphire laser pulse of $12.0 \mathrm{~mJ}$ and 40 fs, our experiment produces an output pulse of $9.4 \mathrm{fs}$ duration with energy $9.1 \mathrm{~mJ}$ (transverse magnetic mode) or $10.0 \mathrm{~mJ}$ (transverse electric mode) in argon, each exhibiting a nice spatial mode. To evaluate such a tapered waveguide, the linear wave propagation theory and the solution to its complex propagation constant are also presented.
\end{abstract}

High energy light pulses down to few optical cycles are key to a wide variety of applications such as optical frequency combs, supercontinuum sources, and generation of attosecond coherent radiation, driving a quest for reliable, stable and cost-efficient lasers and light pulse manipulations [1-3]. On the laser side, the necessary technology has matured; e.g., Ti:Sapphire amplifiers can easily lift the pulse energy to the millijoule-joule level for a pulse duration longer than $20 \mathrm{fs}$ [2]. As such for ultrafast optics community, one of the most interesting challenges concerns how to compress the laser pulse to a desired duration with a nice, focusable spatial mode and a high energy throughput.

To date, there are several compression schemes which lead to pulse durations down to the few-cycle regime. Traditionally, hollow core fibers [4] and femtosecond filamentation [5] are commonly-used methods for extreme pulse compression below $5 \mathrm{fs}$, but with the output energy mostly confined within the submillijoule level mainly due to material damage or intensity clamping. For the former scheme, to increase the output energy, there has been significant effort devoted to steering clear of the onset of gas ionization as the pulse experiences self-phase modulation (SPM) in gas; e.g., Bohman and co-workers realized generation of $5 \mathrm{fs}, 5 \mathrm{~mJ}$ pulses in helium [6] by applying an increasing pressure gradient along the hollow fiber [7]. Noteworthily, the optical parametric chirped-pulse amplification (OPCPA) technique [8] seems to provide a promising route to the intensity scaling problem, but it requires another pump laser and an elaborate setup. By contrast, a recently proposed hollow planar waveguide (PWG) scheme $[9,10]$, regardless of its intrinsic leaky-mode nature $[11,12]$, defines an interesting compromise between pulse compression and energy throughput. But the spatialmode quality is still a critical issue which entails lowering the gas pressure for pulse compression [13].

In this article, we propose a hollow tapered planar waveguide (tPWG) made flared forwards [14] for compres- sion of high-energy ultrashort laser pulses, primarily motivated by a recent technology focus which claimed that a tapered waveguide triumphs over the ridge and stripe ones when designed for high-power diode lasers [15]. This innovative idea, although less frequently mentioned before, indeed has its origin; As early as 1965, Vogel used tapered dielectric tubes and rods for transmission of highpower laser light [16]. Here we demonstrate that, when applying to high-energy ultrashort pulse compression, such a flared-taper configuration has the merit of combining the high output powers with the high beam-quality previously not-easy-attainable in a conventional PWG (cPWG, for later distinction), thereby enabling the pulse duration to be shorter than can be expected.

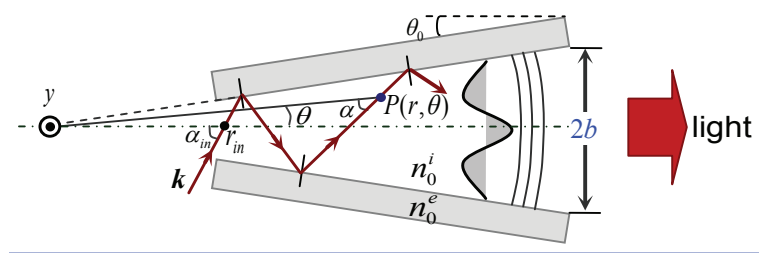

FIG. 1. (Color online) Schematic showing the ray trace (red line) in a flared-taper configuration consisting of two slightly tilted polished fused-silica slabs.

As a starting point, we consider linear wave propagation in a tPWG as sketched in Fig. 1, which consists of two polished fused-silica slabs with $2 a$ the gap width at the entrance and with $2 b$ at the exit. Let $r$ and $\theta$ be the polar coordinates in the plane of incidence and assuming there to be no component of the field varying in the direction perpendicular to the plane of incidence (say, $y$-direction). Therefore, the propagation of e.m. radiation at the carrier wavelength $\lambda$ in such a waveguide can be described by the Helmholtz equation [17]

$$
\nabla^{2} \psi+k^{2} \psi=0
$$


where $\psi(r, \theta)$ is the $y$-component of the electric field or of the magnetic field, corresponding to the transverse electric (TE) mode or transverse magnetic (TM) mode situation, $\nabla^{2}$ is the Laplace operator in $(r, \theta)$, and $k=n_{0} k_{0}$ is the wave number in medium with index $n_{0}=n_{0}^{i}$ in the gap and $n_{0}=n_{0}^{e}$ in the slabs $\left(k_{0}=2 \pi / \lambda\right)$.

We note that, if the field does not penetrate into the confining plate material, i.e., vanishes at the boundary $\theta=$ $\pm \theta_{0}$ ( $\theta_{0}$ is the tilt angle of the plate), Eq. (1) has exact eigenmode solutions, given by [18]

$$
\psi_{m}(r, \theta)=H_{\kappa}^{(1)}\left(n_{0}^{i} k_{0} r\right) \cos \left(\kappa \theta-\varphi_{m}\right),
$$

where $H_{\kappa}^{(1)}$ is the Hankel function of the first kind of (fractional) order $\kappa=(m+1) \pi / 2 \theta_{0}$. Here for definiteness, we use even integer $m$ for even TE/TM modes $\left(\varphi_{m}=0\right)$ and odd $m$ for odd TE/TM modes $\left(\varphi_{m}=\pi / 2\right)$ [17]. Under the circumstances, the propagation constant $\beta$ in the $r$ direction, defined through $\psi_{m}(r, \theta)=\Psi(r, \theta) \exp (i \beta r)$ (here $\Psi(r, \theta)$ is the mode amplitude), can be found from the asymptotic expansion of $H_{\kappa}^{(1)}\left(n_{0}^{i} k_{0} r\right)$ in Eq. (2). For our present conditions, we always have $n_{0}^{i} k_{0} r>\kappa \gg 1$ and as a result have an approximation $H_{\kappa}^{(1)}\left(n_{0}^{i} k_{0} r\right) \simeq$ $\exp [i \kappa(\tan \vartheta-\vartheta)-i \pi / 4] / \sqrt{\pi \kappa \tan \vartheta / 2}$, where $\vartheta=$ $\arccos \left(\kappa / n_{0}^{i} k_{0} r\right)$ [19]. Hence, by direct comparison, we obtain

$$
\beta(r) \simeq n_{0}^{i} k_{0}\left[1-\frac{(m+1)^{2} \pi^{2}}{8\left(n_{0}^{i} k_{0} r \theta_{0}\right)^{2}}\right] .
$$

In reality, the field in the confining material is nonzero due to mode leakage $[11,12]$. Hence the propagation constant $\beta$ given by Eq. (3) should be modified, that is, should be complex, so as to reflect the leaky-mode nature. For this end, we use a modified ray-optics approach [17] and solve for the complex propagation constant in a perturbed manner $\beta(r)=\beta^{(0)}(r)+i \delta(r) / 2$ [20], where $\beta^{(0)}$ is the phase delay per unit propagation distance and $\delta$ is the perturbed term accounting for the power loss.

As illustrated in Fig. 1, we assume now that there exists an arbitrary ray of grazing incidence $\left(\alpha_{i n} \ll 1\right)$ which passes through the point $P(r, \theta)$. The ray trace can thus be determined by $r=r_{i n} \sin \left(\alpha_{i n}\right) / \sin (\alpha)$, where $r_{i n}$ is the polar radius of incident ray associated with $\alpha_{i n}$, and $\alpha$ is the angle formed at $P$ point (see Fig. 1). In order to build up a field mode in the waveguide, it is required that the phase shift along the $\hat{\theta}$-direction encountered by the original wave must be an integer multiple of $2 \pi$ radians of that encountered when the wave reflects twice [17]. Therefore we can write the resultant mode condition as

$$
4 r_{i n} \theta_{0} n_{0}^{i} k_{0} \sin \left(\alpha_{i n}\right)=2(m+1) \pi,
$$

where $m$ is an integer defined as in Eq. (3). Notice that each phase shift introduced by the reflection at the interface, which has a value of either 0 or $2 \pi[17,20]$, has been combined into the term on the right-hand side of Eq. (4).
Considering that $\beta^{(0)}(r)=n_{0}^{i} k_{0} \cos (\alpha)$, it follows readily from the above mode condition, to first order, that

$$
\beta^{(0)}(r) \simeq n_{0}^{i} k_{0}\left[1-\frac{(m+1)^{2} \pi^{2}}{8\left(n_{0}^{i} k_{0} r \theta_{0}\right)^{2}}\right],
$$

which is shown to be the same as the one derived in the nonleaky situation, see Eq. (3).

The attenuation coefficient $\delta$ at $P$ point can be evaluated according to the definition of power loss, namely,

$$
\prod_{j=1}^{J}\left|r_{F}^{(j)}\right|^{2}=e^{-\delta(r)\left(r-r_{i n}\right)},
$$

where $r_{F}^{(j)}$ is the Fresnel's reflection coefficient at the $j$-th reflection and $J \simeq \alpha_{i n}\left(1-r_{i n} / r\right) / 2 \theta_{0}$ is the total number of reflection as the ray reaches the point $P(r, \theta)$. As one can verify, at the condition of grazing incidence $\left(\alpha_{i n} \ll\right.$ 1), the following approximation for $r_{F}^{(j)}$ holds

$$
\ln \left(-r_{F}^{(j)}\right) \simeq-\frac{2 \sin \left[\alpha_{i n}-(2 j-1) \theta_{0}\right]}{\Delta},
$$

where $\Delta=\sqrt{n_{e i}^{2}-1}$ for TE modes and $\Delta=$ $\sqrt{n_{e i}^{2}-1} / n_{e i}^{2}$ for TM modes, with $n_{e i}=n_{0}^{e} / n_{0}^{i}>1$. Then, by use of Eqs. (4), (6), and (7), we find that

$$
\delta(r) \simeq \frac{(m+1)^{2} \pi^{2}}{4\left(n_{0}^{i} k_{0} r \theta_{0}\right)^{2} \Delta} \frac{a+r \theta_{0}}{a^{2}} .
$$

As a result, by combining Eqs. (5) and (8) into $\beta(r)$, the complex propagation constant for either TE or TM leaky mode follows readily

$\beta(r) \simeq n_{0}^{i} k_{0}\left[1-\frac{(m+1)^{2} \pi^{2}}{8\left(n_{0}^{i} k_{0} r \theta_{0}\right)^{2}}\right]+\frac{i(m+1)^{2} \pi^{2}}{8\left(n_{0}^{i} k_{0} r \theta_{0}\right)^{2} \Delta} \frac{a+r \theta_{0}}{a^{2}}$.

One can prove that if $\theta_{0}$ approaches zero (henceforth $r \theta_{0} \rightarrow a$ ), this leaky-mode constant, Eq. (9), can reduce exactly to the one presented in Refs. [9-11]. Importantly, the attenuation term $\delta(r)$ here provides an estimate of the leaky-mode power loss; e.g., for the $m$-th single mode, the total power loss in units of $\mathrm{dB} / \mathrm{m}$ is $4.34 \delta\left(b / \theta_{0}\right)$. It states that the high-order modes undergo higher power loss than the fundamental one, and that the smaller the waveguide separation is, the more strongly the modes dissipate [11]. Also, due to a large $\Delta$, the TE modes are expected to have a higher throughput, about $7-10 \%$ larger than TM modes.

We verify these predicted results experimentally through our Ti:sapphire chirped pulse amplification system which delivers $12 \mathrm{~mJ}$ pulse energy at $100 \mathrm{~Hz}$ with $40 \mathrm{fs}$ duration. The beam $1 / e^{2}$ radius is $6.8 \mathrm{~mm}$ and initially, the polarization is parallel to the plane of propagation (TM) which can be changed to the y direction (TE) by a halfwave plate. The desired waveguide was constructed using two polished fused-silica slabs $(21 \mathrm{~cm}$ long, $1.9 \mathrm{~cm}$ wide, and $0.6 \mathrm{~cm}$ thick) separated by $102 \mu \mathrm{m}$ thick plastic spacers at the entrance and $127 \mu \mathrm{m}$ at the exit. It was then properly placed in a $1.5 \mathrm{~m}$ long gas cell with two $0.5 \mathrm{~mm}$ 
thick antireflection-coated fused-silica windows. We use one cylindrical mirror of $0.75 \mathrm{~m}$ focal length to focus the beam tightly into the entrance of the waveguide and use another one of $1.0 \mathrm{~m}$ focal length to collimate the exiting beam. The output energy was measured to be $9.1 \mathrm{~mJ}$ for TM mode and $10.0 \mathrm{~mJ}$ for TE mode, resulting in an energy throughput of $76 \%$ and $83 \%$ respectively, each nearly $8 \%$ smaller than our analytical predictions based on Eq. (8). We argue that this slight discrepancy is mainly due to the realistic imperfect coupling efficiency $(<100 \%)$ occurred during launching the incident beam into the waveguide entrance. Besides, the reason may lie in that the realistic nonlinear propagation $[14,21]$, to some degree, limits the energy throughput, leading to the measurements deviating from the linear theory above. However, our recent companion work [13], which provided the detailed discussions on the linear and nonlinear propagation, shows that the waveguide throughput is not a strong function of the gas pressure (thus, the nonlinearity), provided that the interaction regime is below strong ionization, what is certainly true in our conditions. Therefore, a linear theory given here can be thought to be sufficient in order to discuss the throughput.

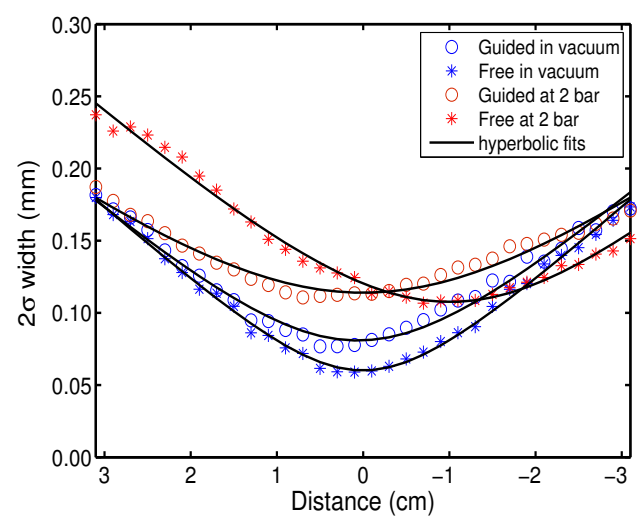

FIG. 2. (Color online) Second moment ( $2 \sigma)$ widths of the beam near the geometrical focus measured in vacuum and at 2 bar for a TM mode along the guided or free dimension, respectively, all fitted by hyperbolic curves.

Nonetheless, despite that we ascribe a high energy throughput to our tapered waveguide design [16], an intriguing question arises as to whether such a flared-taper configuration could at the same time improve the beam quality as compared to the cPWG with a constant separation, $\sim(a+b)$. To address this question, it requires that the focusability of the emerging beam be measured under different gas pressures in the waveguide (here we use argon as the filling gas) [13]. We performed this experiment by, first, weakening the exiting beam subsequently using the reflections of three fused-silica wedges, then focusing it by a 1-m focal length lens directly onto the chip of a CCD camera (uEye UI-2210-M) placed movable along the optical axis, and as a final step, recording the spot images in different positions close to the geometrical focus from which post-processing data were obtained.

Figure 2 illustrates the second moment $(2 \sigma)$ widths of the beam (taking TM mode for our present purpose) measured in vacuum and at 2 bar of argon, respectively, exhibiting a good agreement with their hyperbolic fits [22] along either the guided or free dimension of the waveguide. Based on these hyperbolic fitting data, one can readily obtain their beam propagation factor, $M^{2}$, an important parameter usually used to characterize the beam quality. Table I summarizes the results, including those obtained at 1 bar and 1.6 bar of argon, as well as those measured in a $127 \mu \mathrm{m}$-separated cPWG under otherwise identical conditions (see the right two columns). Here for convenience of comparison, all these values are normalized against the $M^{2}$-values of the input laser along the corresponding directions, which were measured at atmosphere pressure to be $M_{\imath \mathrm{g}}^{2}=1.89$ and $M_{\imath \mathrm{f}}^{2}=1.34$. Here the subscripts $g$ and $f$ denotes the $M^{2}$-values measured along the guided and free dimensions, respectively, and the subscript $\imath$ is specially used for the input beam just delivered by our Ti:sapphire laser. It is clearly seen that the beam quality deteriorates unavoidably as the pressure grows because of small-scale self-focusing [21], and worse along the free dimension than along the guided one. However, compared to the cPWG configuration, our tPWG introduces much smaller adverse effect on the beam quality along each dimension, particularly along the guided dimension. It is especially interesting to note that for the tPWG design, despite the increasing gas pressure, the beam quality along the guided dimension remains almost as good as in vacuum (The ratio $M_{\mathrm{g}}^{2} / M_{\imath \mathrm{g}}^{2}$ ranges from 0.87 in vacuum to 1.05 at 2 bar). The ratio below unity in vacuum and 1 bar suggests the spatial filtering of the tPWG which tends to clean the higher-order modes excited in the high-power laser. All this is not surprising because a flared taper was proved to enable high beam quality and high power throughput simultaneously [15].

Note that the deteriorative process developed in the free dimension is unavoidable for all waveguide-based highenergy pulse compressions $[9,10]$. The complete explanation of it should be within the context of the generalized (3+1)D nonlinear Schrödinger equation including the higher-order dispersion and nonlinearity effects such as the third-order dispersion, plasma defocusing, and multiphoton ionization [21]. For detailed discussions, one can refer to our recent paper [13] and here we will not go further on this issue.

At last, we measured the intensity and phase of the compressed pulse (TM mode) by using a single-shot frequencyresolved optical gating (FROG) [23]. The spectral phase was compensated by two pairs of chirped mirrors ( $\varnothing 45$ $\mathrm{mm})$ with total group-delay dispersion of $-400 \mathrm{fs}^{2}$ (8 bounces). The third-order dispersion was optimized by using an acousto-optic programmable filter-Dazzler [13]. The temporal intensity and phase are shown in Fig. 3, mea- 
TABLE I. Normalized $M^{2}$-values of the emerging beam (TM mode) measured at different pressures of argon for two types of waveguides.

\begin{tabular}{lcccc}
\hline \hline & \multicolumn{2}{c}{ tPWG } & \multicolumn{2}{c}{$\mathrm{cPWG}$} \\
Pressure (in bar) & $M_{\mathrm{g}}^{2} / M_{\imath \mathrm{g}}^{2}$ & $M_{\mathrm{f}}^{2} / M_{\imath \mathrm{f}}^{2}$ & $M_{\mathrm{g}}^{2} / M_{\imath \mathrm{g}}^{2}$ & $M_{\mathrm{f}}^{2} / M_{\imath \mathrm{f}}^{2}$ \\
\hline vacuum & 0.87 & 0.95 & 1.11 & 1.06 \\
1.0 & 0.95 & 1.23 & 1.30 & 1.38 \\
1.6 & 1.02 & 1.46 & 1.46 & 1.64 \\
2.0 & 1.05 & 1.68 & - & - \\
\hline \hline
\end{tabular}

sured at the center of the beam and at 2 bar of argon. The measured spatial mode scaled on the millimeter paper (left inset) and the FROG trace taken by a near-UV high resolution digital camera (right inset) are also presented there. It is shown that with a pressure of 2 bar, a pulse intensity full width at half maximum (FWHM) as low as 9.4 fs was obtained, without introducing too much modulation in the spatial mode. We point out that for the TE mode, the same amount of compression can be achieved with a pressure of 1.8 bar.

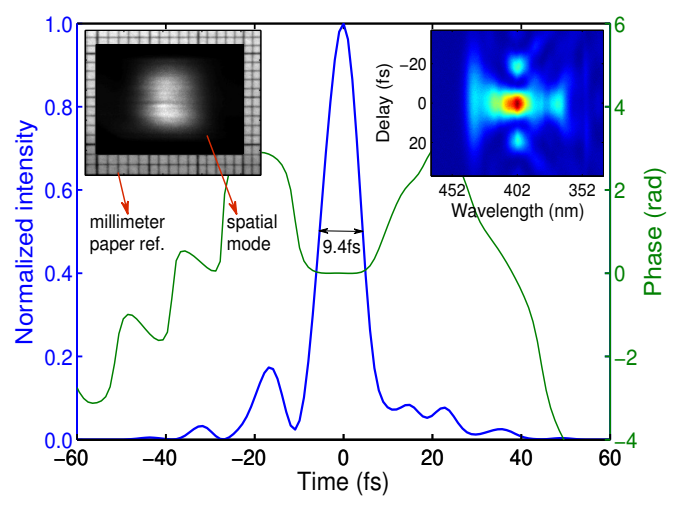

FIG. 3. (Color online) Intensity profile and phase of the compressed pulse measured at 2 bar of argon, with the spatial mode and FROG trace being shown in the left and right insets, respectively.

In summary, a hollow tapered planar waveguide was put forward for use in high-energy ultrashort pulse compressions, with results suggesting a very good trade-off among the energy throughput, the beam focusability, and the pulse compressibility. With our Ti:sapphire laser of $12 \mathrm{~mJ}$ and $40 \mathrm{fs}$, we obtain an output pulse of energy up to $10 \mathrm{~mJ}$ and duration as low as 9.4 fs FWHM with a nice spatial mode. This corresponds to a reliable terawatt ultrashort table-top laser source, a necessary tool for probing the dynamics of plasmas in the relativistic intensity regime [24].

This work was partially supported by the DGA through Grant No. 200795091(A.M.), the National Science Foundation of China through Grant No. 10874024 (S.C.), the Qing Lan Project of Jiangsu Province (S.C.), and the Deutsche Akademie der Naturforscher Leopoldina through
Grant No. BMBF-LPD 9901/8-181 (C.L.A.).

* Electronic mail: andre.mysyrowicz@ensta.fr

[1] F.X. Kärtner (Ed.), Few-Cycle Laser Pulse Generation and Its Applications (Springer, Berlin, 2004).

[2] U. Keller, "Recent developments in compact ultrafast lasers," Nature 424, 831-838 (2003).

[3] F. Krausz and M. Ivanov, "Attosecond physics,” Rev. Mod. Phys. 81, 163-234 (2009).

[4] M. Nisoli, S. De Silvestri, O. Svelto, R. Szipöcs, K. Ferencz, Ch. Spielmann, S. Sartania, and F. Krausz, "Compression of high-energy laser pulses below 5 fs," Opt. Lett. 22, 522-524 (1997).

[5] C.P. Hauri, W. Kornelis, F.W. Helbing, A. Heinrich, A. Couairon, A. Mysyrowicz, J. Biegert and U. Keller, "Generation of intense, carrier-envelope phase-locked few-cycle laser pulses through filamentation," Appl. Phys. B 79, 673677 (2004).

[6] S. Bohman, A. Suda, T. Kanai, S. Yamaguchi, and K. Midorikawa, "Generation of $5.0 \mathrm{f} \mathrm{s}, 5.0 \mathrm{~mJ}$ pulses at $1 \mathrm{kHz}$ using hollow-fiber pulse compression,” Opt. Lett. 35, 18871889 (2010).

[7] A. Suda, M. Hatayama, K. Nagasaka, and K. Midorikawa, "Generation of sub-10-fs, 5-mJ-optical pulses using a hollow fiber with a pressure gradient," Appl. Phys. Lett. 86, 111116 (2005).

[8] N. Ishii, L. Turi, V.S. Yakovlev, T. Fuji, F. Krausz, A. Baltuska, R. Butkus, G. Veitas, V. Smilgevicius, R. Danielius, and A. Piskarskas, "Multimillijoule chirped parametric amplification of few-cycle pulses," Opt. Lett. 30, 567-569 (2005).

[9] J. Chen, A. Suda, E.J. Takahashi, M. Nurhuda, and K. Midorikawa, "Compression of intense ultrashort laser pulses in a gas-filled planar waveguide," Opt. Lett. 33, 2992-2994 (2008).

[10] S. Akturk, C.L. Arnold, B. Zhou, and A. Mysyrowicz, "High-energy ultrashort laser pulse compression in hollow planar waveguides," Opt. Lett. 34, 1462-1464 (2009).

[11] D.B. Hall and C. Yeh, "Leaky waves in a heteroepitaxial film,” J. Appl. Phys. 44, 2271-2274 (1973).

[12] J. Hu and C.R. Menyuk, 'Understanding leaky modes: slab waveguide revisited," Adv. Opt. Photon. 1, 58-106 (2009).

[13] C.L. Arnold, B. Zhou, S. Akturk, S. Chen, A. Couairon, and A. Mysyrowicz, "Pulse compression with planar hollow waveguides: a pathway towards relativistic intensity with table-top lasers,” New J. Phys. 12, 073015 (2010).

[14] K. Hayata, A. Misawa, and M. Koshiba, "Nonlinear beam propgation in tapered waveguides," Electron. Lett. 25, 661662 (1989).

[15] N. Anscombe, "Tapered triumph," Nature Photon. 3, 24-25 (2009).

[16] K. Vogel, "Transmission of high-power laser light through tapered dielectric tubes and rods," Nature 207, 281-282 (1965).

[17] K. Iizuka, Elements of Photonics, Vol. II (Wiley, New York, 2002), Chapt. 9.

[18] C. Bergemann, H. Keymeulen, and J.F. van der Veen, "Focusing X-Ray Beams to Nanometer Dimensions," Phys. 
Rev. Lett. 91, 204801 (2003).

[19] I.S. Gradshteyn and I.M. Ryzhik, Table of Integrals, Series, and Products (Elsevier, Amsterdam, 2007), Chapt. 8.4.

[20] A. Yariv and P. Yeh, Optical Waves in Crystals (Wiley, New Jersey, 2003), Chapt. 11.

[21] A. Couairon and A. Mysyrowicz, "Femtosecond filamentation in transparent media," Phys. Rep. 441, 47-189 (2007).

[22] W. Riede and W. Mayerhofer, "Beam quality of a highpower $\mathrm{CO}_{2}$ laser MOPA system," Optics \& Laser Technology 30, 491-496 (1998).
[23] S. Akturk, C. D’Amico, and A. Mysyrowicz, "Measuring ultrashort pulses in the single-cycle regime using frequencyresolved optical gating," J. Opt. Soc. Am. B 25, A64-A69 (2008).

[24] D. Umstadter, S.-Y. Chen, A. Maksimchuk, G. Mourou, and R. Wagner, "Nonlinear optics in relativistic plasmas and laser wakefield acceleration of electrons," Science 273, 472475 (1996). 